\title{
Caracterización psicosocial y criminológica de adolescentes chilenos con prácticas abusivas sexuales
}

\author{
Miguel Salazar-Muñoz, Mg. ${ }^{a}$ \\ Universidad San Sebastián, Chile \\ Luis Álvarez-Vivar, Mg. ${ }^{\mathrm{b}}$ \\ Universidad Austral de Chile, Chile
}

\author{
Yonatan Bustamante-Cárcamo, Mg. ${ }^{c}$ \\ Universidad Santo Tomás, Chile \\ Ricardo Pérez-Luco Arenas, Ph.D.d \\ Universidad de La Frontera, Chile
}

miguel.salazar@uss.cl

\section{Resumen (analítico)}

El objetivo de este estudio fue caracterizar las variables psicosociales y criminológicas asociadas a los adolescentes que desarrollan prácticas abusivas sexuales (PAS), en función de la edad de las víctimas. Mediante un diseño no experimental y transversal, con características descriptivas y correlacionales, se analizan antecedentes psicosociales y criminológicos de 232 adolescentes varones que han realizado PAS. Los resultados indican diferencias estadísticas significativas entre los adolescentes que cometen PAS contra víctimas menores, en contraste con quienes abusan de víctimas pares, tanto en las variables psicosociales (trayectoria escolar e historial de victimizaciones) como en las variables criminológicas (contexto de la práctica abusiva sexual y relación adolescente-víctima). Finalmente, se discuten los resultados en relación con la evidencia internacional y las implicaciones de la generación de conocimientos sobre la temática.

\section{Palabras clave}

Ofensa sexual, víctima, adolescencia, comportamiento social, violencia

\section{Thesauro}

Tesauro de Ciencias Sociales de la Unesco.
Para citar este artículo

Salazar-Muñoz, M., Álvarez-Vivar, L., BustamanteCárcamo, Y., \& Pérez-Luco Arenas, R. (2021). Caracterización psicosocial y criminológica de adolescentes chilenos con prácticas abusivas sexuales. Revista Latinoamericana de Ciencias Sociales, Niñez y Juventud, 19(1), 1-25. https://dx.doi.org/10.11600/rlcsnj.19.1.4645

\section{Historial}

Recibido: 19.03.2020

Aceptado: 02.07.2020

Publicado: 09.12.2020

\section{Información artículo}

El presente artículo se inserta dentro del proyecto de investigación Evaluación de adolescentes con prácticas abusivas sexuales desde el Modelo Multidimensional de Intervención Diferenciada con Adolescentes (MMIDA $\left.{ }^{\odot}\right)^{\prime \prime}$ desarrollado entre los años 2014-2017 por programa de Magister en Psicología Jurídica y Forense de la Universidad de La Frontera, en el marco del proyecto FONDEF D08i-1205. Área: Ciencias Sociales. Subárea: Interdisciplinaria. 


\section{Psychosocial and criminological characterization of Chilean adolescents with sexually abusive behavior}

Abstract (analytical)

The aim of this study is to characterize the psychosocial and criminological variables associated with adolescents who develop sexually abusive behavior, depending on the age of the victims. Psychosocial and criminological data were collected from 232 male adolescents who have demonstrated sexually abusive behavior. This data was analyzed using a non-experimental, cross-sectional design with descriptive and correlational characteristics. The results indicate statistically significant differences between adolescents who offended against a child victim versus adolescents who offended against a peer-aged victim, both in psychosocial variables (school history and history of victimization) and criminological variables (context of sexually abusive behavior and adolescent-victim relationship). The results are discussed in relation to international evidence and the implications of generating knowledge in this area.

Keywords

Sexual offense, victim, adolescence, social behavior, violence.

\section{Caracterização psicossocial e criminológica de adolescentes chilenos com comportamentos sexuais abusivos}

Resumo (analítico)

O objetivo deste estudo foi caracterizar as variáveis psicossociais e criminológicas associadas a adolescentes que desenvolvem comportamentos sexuais abusivos, dependendo da idade das vítimas. A partir de um delineamento transversal não experimental com características descritivas e correlacionais, foi analisado o histórico psicossocial e criminológico de 232 adolescentes do sexo masculino que praticaram comportamento sexual abusivo. Os resultados indicam diferenças estatísticas significativas entre os adolescentes que cometem abuso sexual contra menores e os que cometem contra vítimas de idade semelhante, tanto em variáveis psicossociais (histórico escolar e histórico de vitimizações) quanto em variáveis criminológicas (contexto de comportamento sexual abusivo e relacionamento adolescente-vítima). Por fim, os resultados são discutidos em relação às evidências internacionais e as implicações da geração de conhecimentos na área.

Palavras-chave

Ofensa sexual, vítima, adolescência, comportamento social, violência.

Información autores

[a] Psicólogo, Universidad Austral de Chile. Magíster en Psicología Jurídica y Forense, Universidad de La Frontera. Doctorando en Psicología, Salud y Calidad de Vida, Universidad de Gerona. Docente Facultad de Psicología, Universidad San Sebastián, Chile. H5: 2. iD 0000-0002-0294-1395. Correo electrónico: miguel.salazar@uss.cl [b] Psicólogo, Universidad Austral de Chile. Magíster en Psicología Jurídica y Forense, Universidad de La Frontera. Docente Instituto de Psicología, Universidad Austral de Chile. H5: 3. iD 0000-0002-0429-7337. Correo electrónico: luis.alvarez@uach.cl

[c] Psicólogo, Universidad Austral de Chile. Magíster en Psicología Jurídica y Forense, Universidad de La Frontera. Doctorando en Psicología, Universidad del País Vasco. Docente Carrera de Psicología, Facultad de Ciencias Sociales y Comunicación, Universidad Santo Tomás, Chile. Director de ONG Coincide. H5: 2. ID 0000-0002-3597-8856. Correo electrónico: ybustamante2@santotomas.cl

[d] Psicólogo, Universidad de La Frontera. Magíster en Ciencias Sociales y Políticas, Flacso, México. Doctor en Psicología, Universidad Pontificia de Salamanca, España. Profesor Asociado Departamento de Psicología, Universidad de La Frontera. H5: 8. iD 0000-0002-7051-5005. Correo electrónico: ricardo.perez-luco@ufrontera.cl 


\section{Introducción}

$\mathrm{E}_{\text {cas abusivas sexuales (en adelante PAS) ha cobrado notoriedad, hasta transfor- }}$ marse en un foco de análisis para diferentes áreas de estudio, tales como las ciencias sociales, las ciencias jurídicas y la salud pública. Los estudios de caracterización han informado que estos adolescentes son en su mayoría hombres, de entre 12 y 19 años, responsables de alrededor del $20 \%$ de las violaciones y del $50 \%$ de los casos de abuso sexual cometidos cada año en Estados Unidos (Finkelhor et al., 2009). Si bien estas cifras pueden variar de acuerdo con los sistemas judiciales y estadísticos de cada país, existe una tendencia a establecer que, por cada 1000 individuos de una cohorte de nacimiento, entre 3 y 5 son arrestados por un delito sexual durante la adolescencia (Lussier, 2017).

En la actualidad, se ha generado suficiente evidencia para demostrar que los adolescentes vinculados a PAS constituyen un grupo heterogéneo, es decir, difieren entre sí en cuanto a variables individuales, familiares y comunitarias (Cale et al., 2016; Everhart et al., 2019; Lillard et al., 2019; Malvaso et al., 2020; Ryan \& Otonichar, 2016; Ueda, 2017; Wanklyn et al., 2012; Worling \& Langton, 2012), lo que, sumado a los vertiginosos cambios de la adolescencia, dificulta la investigación, evaluación y tratamiento de esta problemática (Chaffin, 2008; Hunter et al., 2003; Oxnam \& Vess, 2008). Con el fin de reducir esta complejidad y de aportar a la especialización de los tratamientos, se han emprendido varias investigaciones para delimitar distintos subgrupos de adolescentes con PAS, mediante la utilización de variables psicosociales y criminológicas aplicadas tanto a los agresores como a las víctimas y a las PAS cometidas (Aebi et al., 2012).

Entre las diversas variables utilizadas para clasificar a los adolescentes con PAS, una de las que ha propiciado más consenso y ha evidenciado una mayor consistencia empírica es la edad de las víctimas (Keelan \& Fremouw, 2013). Estos estudios se han concentrado en países de Norteamérica (Fanniff \& Kolko, 2012; Leroux et al., 2016; Lillard et al., 2019; Short, 2018) y Europa (Aebi et al., 2012; Benedicto et al., 2017; Glowacz \& Born, 2013; 
Gunby \& Woodhams, 2010; Stevens et al., 2013), en donde, por lo general, se han empleado tres subgrupos para clasificar este comportamiento en los adolescentes: a) con víctimas menores (VM); b) con víctimas pares-adultas (VPA); y c) con víctimas mixtas (VMI).

Aunque estas clasificaciones han permitido profundizar en la especificidad del análisis de los adolescentes con PAS, la investigación se encuentra aún en pleno desarrollo (Brown, 2019). Algunas limitaciones para avanzar hacia perfiles validados a través de este tipo de estudios han sido planteadas por Keelan y Fremouw (2013) quienes, después de realizar un análisis crítico de la literatura disponible, concluyeron que la posibilidad de hacer comparaciones entre los estudios y de obtener conclusiones a partir de estos se ve obstaculizada por diversas dificultades metodológicas; por ejemplo, por las definiciones inconsistentes de las variables usadas, la baja potencia estadística de las muestras y la falta de medidas estandarizadas para su medición.

Pese a lo descrito, y de acuerdo con algunas tendencias, es posible afirmar que los adolescentes con VM son más propensos a ser sumisos, presentan menor autoestima, muestran problemas de conductas internalizantes, un mayor nivel de victimización y aislamiento social; mientras que los adolescentes con VP tienen una tendencia a ser más agresivos durante la PAS y registran una mayor escalada de comportamientos antisociales y conductas externalizantes. Dichos factores motivan el diseño de estrategias de intervención diferenciadas en virtud de las particularidades de los distintos subtipos (Benedicto et al., 2017; Glowacz \& Born, 2013; Lillard et al., 2019; Ueda, 2017).

Para Krause et al. (2020) ambos grupos no manifiestan diferencias significativas en reincidencia sexual; sobre este ítem se observa un promedio de $9.2 \%$ para los adolescentes con PAS-VM (prácticas abusivas sexuales dirigidas a víctimas menores) y de 5.7\% para quienes presentan PAS-VP (prácticas abusivas sexuales dirigidas a víctimas pares). Ahora bien, es sabido que muchos de los adolescentes desisten de cometer delitos sexuales en la adultez (Lussier \& Blokland, 2014) y que las tasas de reincidencia sexual de los adolescentes que llevan a cabo PAS son bajas, pues registran una tasa media ponderada de $2.75 \%$ de reincidencia, según estudios realizados posteriores al año 200o. Este dato resulta auspicioso, debido a que se manifiesta una disminución importante respecto a la tasa del 10.3\%, reportada en los estudios aplicados entre 1980 y 1995, lo que supone un nivel de efectividad potencial de las políticas públicas destinadas a reducir la violencia sexual en la sociedad (Caldwell, 2016).

En Latinoamérica, la investigación sobre adolescentes con comportamientos desadaptativos se ha enfocado mayormente en el análisis de aquellos que cometen delitos contra la 
propiedad o contra las personas (Ávila-Navarrete, 2017; Komatsu \& Bazon, 2015). Así mismo, los estudios sobre los adolescentes que desarrollan PAS son incipientes, en la medida en que se limitan al análisis de registros de denuncias, a estudios preliminares de caracterización y sistematizaciones de experiencias de intervención (Salazar-Muñoz et al., 2016).

En el caso de Chile, estudios enmarcados en la criminología evolutiva han profundizado en el conocimiento sobre las trayectorias de adolescentes infractores, entre ellos, adolescentes con PAS. Al respecto, Alarcón et al. (2017), tras evaluar a 571 adolescentes chilenos que cumplían una sanción en un programa de responsabilidad penal, identificaron dentro de los tipos de delincuencia adolescente uno al que denominaron delincuencia compleja, correspondiente a un grupo de adolescentes con características heterogéneas y con un comportamiento delictivo no reiterativo en el tiempo, manifestado en contextos sociales adaptativos. Además, en gran medida dicho comportamiento estaba influenciado por trastornos de salud mental, alteraciones en la personalidad o problemas psicológicos asociados a haber vivido o experimentado experiencias traumáticas, entre las cuales se halla el subtipo de adolescentes con PAS (Pérez-Luco et al., 2017).

Con relación a la magnitud del fenómeno en Chile, según datos del Ministerio Público (2019), las denuncias por delitos sexuales cometidos por adolescentes han sufrido un significativo aumento; durante el año 2019 se alcanzó el mayor número de delitos ingresados (1830) desde la implementación de la Ley de Responsabilidad Penal Adolescente en el año 2007, lo que representa el $4.48 \%$ del total de denuncias por delitos sexuales en el país. Pese a que la cifra parece ser discreta en relación con el total de delitos sexuales, el impacto social generado por la violencia sexual, así como la permeabilidad terapéutica de los adolescentes, justifican la intervención temprana con el ánimo de evitar la cronificación de estos comportamientos (Jenkins et al., 2020).

Por lo anterior, queda en evidencia, en primer lugar, la importancia de avanzar hacia la diferenciación de subtipos de adolescentes que desarrollan PAS, para apoyar la prevención de esta conducta y su tratamiento (ter Beek et al., 2018); en segundo lugar, la ausencia de estudios en Latinoamérica sobre este grupo específico de adolescentes que contribuyan a la fundamentación de orientaciones técnicas que guíen los programas de tratamiento chilenos. En efecto, según lo que se ha demostrado, los modelos generalistas no son adecuados para explicar las PAS, mientras que una perspectiva especializada abre oportunidades para ajustar herramientas de evaluación y enfoques de tratamiento, de acuerdo con las características de cada adolescente (Malvaso et al., 2020; Seto \& Lalumière, 2010). De esta manera, resulta pertinente preguntarse: jes posible notar diferencias 
entre adolescentes chilenos que han perpetrado PAS en contra de VM y aquellos que han agredido a VP? Para ello, el presente estudio se propuso como objetivo caracterizar las variables psicosociales y criminológicas asociadas a adolescentes con PAS, en función de la edad de las víctimas.

\section{Método}

\section{Diseño}

La presente investigación consistió en un estudio de tipo no experimental, transversal, con características descriptivas y correlacionales, en el cual se compararon variables psicosociales y criminológicas de dos muestras de adolescentes que cometieron PAS, quienes fueron clasificados en subgrupos, según la edad de sus víctimas.

\section{Participantes}

A través de un muestreo no probabilístico intencionado se accedió a una muestra de 232 adolescentes varones, quienes cumplieron con los cuatro criterios de inclusión planteados para esta investigación: a) haber cometido alguna PAS; b) tener entre 12 y 18 años; c) estar vigente en alguno de los programas ambulatorios de intervención especializada para PAS; y d) haber culminado el proceso de diagnóstico inicial.

Luego se recogieron los datos de los adolescentes que se encontraban en fase de tratamiento en programas ambulatorios de intervención especializada en PAS propuestos por el Servicio Nacional de Menores (Sename) en ocho regiones del país (Arica y Parinacota, Tarapacá, Antofagasta, Atacama, Metropolitana, Biobío, La Araucanía, Los Lagos) entre los años 2014 y 2015.

Para dar respuesta al objetivo planteado, y con base en criterios teóricos, se conformaron dos grupos: a) adolescentes que habían agredido a víctimas menores (PAS-VM) y b) adolescentes que habían agredido a víctimas pares (PAS-VP); dado que en la muestra no se registraron adolescentes con víctimas adultas, no se tuvo en cuenta este subgrupo. A su vez, para establecer el subgrupo de VM se consideró una diferencia de edad entre la víctima y el ofensor de al menos cuatro años, de manera similar a los estudios anteriores (Fanniff \& Kolko, 2012; Lillard et al., 2019).

Respecto a los dos grupos, no existieron diferencias estadísticas significativas a nivel sociodemográfico, por lo que ambas muestras tienden a manifestarse de forma homogé- 
nea en este tipo de variables. A continuación, se describen las principales características sociodemográficas de los grupos:

- Grupo PAS-VM: conformado por 170 adolescentes, con una edad promedio de 14 años al momento del ingreso al programa. El 56.47\% se encuentra en el estrato socioeconómico medio, el $41.76 \%$ pertenece al bajo y solo el $1.77 \%$ está en el estrato alto. El $88.82 \%$ es de origen urbano y el $85.29 \%$ declara no pertenecer a ninguna etnia indígena. En cuanto a la escolaridad, el $3.5 \%$ ha desertado.

- Grupo PAS-VP: integrado por 62 adolescentes, con una edad promedio de 14.8 años al ingresar al programa. El 66.13\% se halla en el estrato socioeconómico bajo, el $30.65 \%$ en el medio y solo el $3.22 \%$ en el estrato alto. El $79.03 \%$ es de origen urbano y el $88.71 \%$ declara no pertenecer a ninguna etnia indígena. Respecto a la escolaridad, el 19.4\% registra deserción escolar.

\section{Procedimiento}

En primer lugar, mediante una estrategia de investigación formal se obtuvieron las autorizaciones correspondientes de Sename y de los Organismos Colaboradores Acreditados que ejecutan programas de tratamiento para PAS. Posteriormente, los investigadores establecieron contacto directo con los profesionales de cada programa, a fin de proceder con el levantamiento inicial de la información. El procedimiento estuvo constituido por las siguientes fases:

- Fase inicial de implementación: el equipo investigador realizó una jornada de capacitación de $3^{2}$ horas presenciales en la ciudad de Santiago de Chile (Región Metropolitana), cuyos contenidos fueron validados por el Departamento de Psicología de la Universidad de La Frontera. En esta actividad participaron psicólogos y trabajadores sociales de todos los programas de intervención, quienes se capacitaron en los aspectos formales de la investigación, en la correcta selección de la muestra y en la aplicación del instrumento de estudio.

- Fase de seguimiento: el equipo investigador efectuó un acompañamiento y asesoría en terreno a cada uno de los programas de intervención, con énfasis en el análisis de casos y en la revisión del llenado del instrumento, construido por medio de criterios teóricos. Lo anterior permitió cotejar la información y minimizar el sesgo del evaluador. 
- Fase de cierre: el equipo investigador recopiló los antecedentes reunidos por cada programa, para después realizar el análisis de acuerdo con los criterios metodológicos del estudio.

Con el propósito de evitar una posible contaminación de los procesos de tratamiento, los investigadores no tuvieron contacto directo con los adolescentes de la muestra; por lo anterior, la información recabada en el instrumento utilizado se sustenta en el juicio profesional de los responsables de cada caso, quienes completaron el instrumento con base en la información contenida en las carpetas y en los registros individuales de cada adolescente. Antes de ello se obtuvo un consentimiento informado que compromete el resguardo de la identidad, la posibilidad de los participantes de abandonar el estudio en cualquier momento, así como el uso ético de la información y de los resultados.

\section{Instrumentos}

Se utilizó una ficha de caracterización psicosocial y criminológica (F-CPsyC) de construcción empírica basada en dimensiones y criterios señalados en la literatura cientifica disponible (Benedicto et al., 2017; Glowacz \& Born, 2013; Gunby \& Woodhams, 2010; Hendriks \& Bijleveld, 2004; Höing et al., 2010; Keelan \& Fremouw, 2013; Lillard et al., 2019; van der Put \& Asscher, 2015) y validada por el juicio de expertos. La construcción de la F-CPsyC, por parte del grupo investigador, se llevó a cabo tomando en consideración las indicaciones de Muñiz y Fonseca-Pedrero (2019) para la construcción y validación de instrumentos.

La validación inicial de contenido de la F-CPsyC se obtuvo con la opinión informada de una serie de expertos, quienes fueron seleccionados según su amplia trayectoria en docencia e investigación y su experiencia en temáticas afines, tales como la evaluación de adolescentes con comportamiento desadaptativo y con PAS. Igualmente, la validación final de contenido de la F-CPsyC se alcanzó mediante la valoración de múltiples interventores, especializados en el tratamiento de adolescentes con PAS, y siguiendo las recomendaciones propuestas por Escobar-Pérez y Cuervo-Martínez (20o8).

Así, la herramienta final consideró un total de 27 dimensiones, divididas en variables sociodemográficas (5), variables psicosociales (15) y variables criminológicas (7), según la siguiente distribución:

- Variables sociodemográficas: edad, escolaridad, estrato socioeconómico, origen y etnia. 
- Variables psicosociales: trayectoria educativa (promedio de cursos reprobados, problemas escolares y víctima de acoso escolar); antecedentes familiares (estructura familiar, promedio de integrantes familiares, promedio de edad y nivel educativo de adulto responsable); registros de institucionalización (antecedentes de ingresos previos al Sename y promedio de ingresos previos al Sename); y tipo de programas Sename e historial de victimización (promedio de victimizaciones sufridas, observador de violencia intrafamiliar, abuso emocional, abuso físico y sexual).

- Variables criminológicas: edad de PAS, promedio de víctimas, tipo de PAS, contexto de PAS, sexo de la víctima, relación ofensor-víctima e ingresos a programas de responsabilidad penal adolescente por otro tipo de delitos.

\begin{abstract}
Análisis de datos
En primer término, se realizó un análisis exploratorio de datos para describir, resumir y visualizar su naturaleza. Más adelante, se utilizó la estadística descriptiva con el fin de caracterizar las variables sociodemográficas de la muestra total y de las submuestras. Para examinar las diferencias entre los dos grupos, respecto de las variables descritas, se empleó el análisis chi cuadrado $\left(\mathbf{X}^{2}\right)$ de Pearson y la prueba t de Student, mientras que el tamaño del efecto se calculó utilizando la $\mathrm{V}$ de Cramer y la $\mathrm{d}$ de Cohen, respectivamente.
\end{abstract}

\title{
Resultados
}

\section{Perfil psicosocial de los adolescentes que han ejecutado PAS}

\section{Trayectoria educativa}

En la muestra total se destacó que solo el $27.6 \%$ del total de los adolescentes presenta problemas escolares, variable que considera el absentismo escolar y las dificultades de adaptación a los contextos educativos, con un promedio de 1.3 cursos reprobados $(\mathrm{SD}=1.9)$.

Al comparar ambos subgrupos, se encontraron diferencias significativas vinculadas con el hecho de que los adolescentes con PAS-VP presentarían mayores problemas escolares $\left(\mathrm{x}^{2}=23.66, \mathrm{p}<.05\right)$, mayor nivel de reprobación $(\mathrm{t}=1.9, \mathrm{p}<0.05)$ y una menor tasa de acoso escolar $\left(\mathrm{x}^{2}=6.5, \mathrm{p}<.05\right)$ (tabla 1$)$. 


\section{Tabla 1}

Análisis de la trayectoria educativa

\begin{tabular}{|c|c|c|c|c|c|}
\hline Variable & $\begin{array}{c}\text { Total }(n=232) \\
\bar{X} / \%\end{array}$ & $\begin{array}{c}\text { PAS-VM }(n=170) \\
\bar{X} / \%\end{array}$ & $\begin{array}{c}\text { PAS-VP }(n=62) \\
\bar{X} / \%\end{array}$ & $t \circ X^{2}$ & $d \circ V$ \\
\hline Promedio de reprobaciones & 1.3 & 1 & 1.5 & $t=1.04^{\star}$ & 0.43 \\
\hline \multicolumn{6}{|l|}{ Problemas escolares } \\
\hline Sí & 27.6 & 12.9 & 67.7 & \multirow{2}{*}{$X^{2}=23.66^{*}$} & \multirow[t]{2}{*}{0.51} \\
\hline No & 72.4 & 87.1 & 32.3 & & \\
\hline \multicolumn{6}{|l|}{ Víctima de acoso escolar } \\
\hline Sí & 25 & 29.4 & 12.9 & \multirow{2}{*}{$x^{2}=6.5^{\star}$} & \multirow[t]{2}{*}{0.54} \\
\hline No & 75 & 70.6 & 87.1 & & \\
\hline
\end{tabular}

Nota: PAS-VM: prácticas abusivas sexuales dirigidas hacia víctimas menores; PAS-VP: prácticas abusivas sexuales dirigidas a víctimas pares o iguales; * $\mathrm{p}<0.05$.

\section{Antecedentes familiares}

Tal como se observa en la tabla 2, el análisis de la muestra total indica que la composición de las familias de los adolescentes registra un promedio de 4.6 integrantes ( $\mathrm{SD}=1.07)$ y una edad promedio del adulto responsable de 39 años $(S D=1.46)$.

\section{Tabla 2}

Análisis de los antecedentes familiares

\begin{tabular}{|c|c|c|c|c|c|}
\hline Variable & $\begin{array}{c}\text { Total }(n=232) \\
\bar{X} / \%\end{array}$ & $\begin{array}{c}\text { PAS-VM }(n=170) \\
\bar{X} / \%\end{array}$ & $\begin{array}{c}\text { PAS-VP }(\mathrm{n}=62) \\
\bar{X} / \%\end{array}$ & $\operatorname{to} X^{2}$ & dov \\
\hline Promedio integrantes familia & 4.6 & 4.8 & 4.4 & $t=1.07$ & 0.12 \\
\hline \multicolumn{6}{|l|}{ Tipo de familia } \\
\hline Nuclear & 42.24 & 44.12 & 37.1 & & \\
\hline Monoparental & 28.02 & 30 & 22.6 & & \\
\hline Extensa & 9.91 & 9.41 & 11.3 & $X^{2}=7.04$ & 0.13 \\
\hline Compuesta & 17.24 & 14.71 & 24.2 & & \\
\hline Red Sename & 2.59 & 1.76 & 4.8 & & \\
\hline Promedio edad AR & 39 & 37 & 40 & $t=1.46$ & 0.09 \\
\hline \multicolumn{6}{|l|}{ Educación AR } \\
\hline Enseñanza básica & 71.98 & 70.59 & 75.8 & & \\
\hline Enseñanza media & 24.57 & 25.88 & 21 & $X^{2}=0.6267$ & 0.15 \\
\hline Estudios superiores & 3.45 & 3.53 & 3.2 & & \\
\hline
\end{tabular}

Nota: AR: adulto responsable. 
En lo concerniente a la escolaridad, es un hecho llamativo que solo el $24.57 \%$ de los adultos responsables haya completado la enseñanza media y el 3.45\% tenga estudios superiores. La estructura familiar de los adolescentes corresponde a familias nucleares (42.24\%), seguidas de familias monoparentales (28.02\%), compuestas (17.24\%) y extensas (9.91\%). Así mismo, un número menor de adolescentes se encuentra en sistemas de cuidado alternativo del Sename (2.59\%).

A nivel comparativo, no se hallaron diferencias significativas en todas las variables que componen la dimensión familiar. No obstante, desde un punto de vista descriptivo, es posible advertir una ligera elevación en el promedio del número de integrantes del grupo familiar en el hogar de los adolescentes con PAS-VM (4.8), quienes, además, presentan un mayor porcentaje de familias nucleares (44.12\%) y monoparentales (30\%). En contraposición, el grupo conformado por adolescentes con PAS-VP muestra una edad promedio del adulto responsable levemente mayor (40 años), así como mayores porcentajes de estructuras familiares de tipo compuestas (24.2\%), extensas (11.3\%) y red Sename (4.8\%).

\section{Registro de institucionalización}

No se encontraron diferencias significativas entre ambos grupos. Los resultados presentados en la tabla 3 indican que el $84 \%$ de los adolescentes, previo a su ingreso a un programa PAS, mostró ingresos a otros programas del Sename, con un pro-medio de 3.7 programas $(\mathrm{SD}=1.9)$. Los ingresos previos se concentran en programas de diagnóstico (32.3\%), intervención especializada ( $28 \%$ ) y prevención focalizada ( $25 \%$ ), y en menor medida en residencias y familias de acogida (14.7\%). Adicionalmente, solo un $6 \%$ de los adolescentes registró ingresos a programas de responsabilidad adolescente por delitos no sexuales.

\section{Tabla 3}

Análisis de registros de institucionalización

\begin{tabular}{|c|c|c|c|c|c|}
\hline Variable & $\begin{array}{c}\text { Total }(n=232) \\
\bar{X} / \%\end{array}$ & $\begin{array}{c}\text { PAS-VM }(n=170) \\
\bar{X} / \%\end{array}$ & $\begin{array}{c}\text { PAS-VP }(n=62) \\
\bar{X} / \%\end{array}$ & $t \circ X^{2}$ & doV \\
\hline \multicolumn{6}{|l|}{ Ingresos previos Sename } \\
\hline Sí & 84 & 84.7 & 82.3 & \multirow{2}{*}{$X^{2}=0.179$} & \multirow{2}{*}{0.18} \\
\hline No & 16 & 15.3 & 17.7 & & \\
\hline Promedio $n .^{\circ}$ de programas & 3.7 & 2.2 & 4.8 & $\mathrm{t}=1.9$ & 0.17 \\
\hline \multicolumn{6}{|l|}{ Tipo de programa } \\
\hline Diagnóstico & 32.3 & 32.3 & 32.3 & \multirow{4}{*}{$X^{2}=0.40$} & \multirow{4}{*}{0.15} \\
\hline Prevención & 25 & 25.9 & 22.6 & & \\
\hline Especializado & 28 & 27.1 & 30.6 & & \\
\hline Residencia o familia de acogida & 14.7 & 14.7 & 14.5 & & \\
\hline
\end{tabular}

Nota: $\mathrm{p}<0.05$. 


\section{Historial de victimización}

Cabe señalar que la totalidad de los adolescentes ha experimentado distintos tipos de victimizaciones en su trayectoria de vida, con un promedio de 3.5 tipos ( $\mathrm{SD}=1.13$ ). Tal como se muestra en la tabla 4, al analizar la muestra total de adolescentes, los tipos de victimizaciones más observados fueron: haber sido testigo de violencia intrafamiliar (67.7\%), haber experimentado abuso emocional (62.1\%), haber sufrido abuso físico $(56.9 \%)$ y haber vivido un abuso de tipo sexual (53.9\%).

Se encontraron diferencias significativas en dos tipos de victimizaciones, a saber: haber sido testigo de violencia intrafamiliar $\left(\mathrm{x}^{2}=9.97, \mathrm{p}<.05\right)$ y haber experimentado abuso físico $\left(\mathrm{x}^{2}=13.56, \mathrm{p}<.05\right)$. Estas tienden a estar más presentes en los adolescentes con PAS-VM.

\section{Tabla 4}

Análisis del historial de victimizaciones

\begin{tabular}{|c|c|c|c|c|c|}
\hline Variable & $\begin{array}{c}\text { Total }(\mathrm{n}=232) \\
\bar{X} / \%\end{array}$ & $\begin{array}{c}\text { PAS-VM }(n=170) \\
\bar{X} / \%\end{array}$ & $\begin{array}{c}\text { PAS-VP }(n=62) \\
\bar{X} / \%\end{array}$ & $\operatorname{to} X^{2}$ & doV \\
\hline Promedio . $^{\circ}$ de victimizaciones & 3.5 & 3.6 & 3.4 & $t=1.13$ & 0.16 \\
\hline \multicolumn{6}{|l|}{ Tipo de victimizaciones } \\
\hline \multicolumn{6}{|l|}{ Testigo de VIF } \\
\hline Sí & 67.7 & 73.5 & 51.6 & \multirow{2}{*}{$x^{2}=9.97^{*}$} & \multirow{2}{*}{0.52} \\
\hline No & 32.3 & 26.5 & 48.4 & & \\
\hline \multicolumn{6}{|l|}{ Abuso emocional } \\
\hline Sí & 62.1 & 64.7 & 54.8 & \multirow{2}{*}{$x^{2}=1.86$} & \multirow{2}{*}{0.25} \\
\hline No & 37.9 & 35.3 & 45.2 & & \\
\hline \multicolumn{6}{|l|}{ Abuso físico } \\
\hline Sí & 56.9 & 64.1 & 37.1 & \multirow{2}{*}{$X^{2}=13.56^{*}$} & \multirow{2}{*}{0.43} \\
\hline No & 43.1 & 35.9 & 62.9 & & \\
\hline \multicolumn{6}{|l|}{ Abuso sexual } \\
\hline Sí & 53.9 & 54.1 & 53.2 & \multirow{2}{*}{$X^{2}=0.015$} & \multirow{2}{*}{0.18} \\
\hline No & 46.1 & 45.9 & 46.8 & & \\
\hline
\end{tabular}

Nota: VIF: violencia intrafamiliar; ${ }^{*} \mathrm{p}<0.05$ 


\section{Características criminológicas de los adolescentes que han ejecutado PAS}

\section{Edad de las PAS y promedio de víctimas}

Para la muestra total, los resultados expuestos en la tabla 5 evidencian que la edad promedio en la que los adolescentes ejecutan las PAS corresponde a los 13.3 años (DS=3.2), es decir, en la etapa de la adolescencia temprana. Respecto al número de víctimas, el promedio de víctimas para la muestra general asciende a 1.3 (DS=1.5).

Ambas variables no registran diferencias significativas al ser comparadas en los dos grupos. Desde una perspectiva descriptiva, se puede afirmar que los adolescentes con PAS-VP exhibirían una edad ligeramente menor al momento de realizar el evento abusivo (13.1), en comparación con el grupo con PAS-VM, quienes en promedio tienen 13.5 años $(\mathrm{SD}=1.9)$. Entretanto, los adolescentes con PAS-VP cuentan con un promedio superior de víctimas (1.5), con relación a los adolescentes con PAS-VM (1.1).

\section{Tipo de PAS}

A nivel general, el tipo de PAS más recurrente corresponde al abuso sexual infantil (78\%), seguido de violación (12.1\%) y, en menor grado, exposición a pornografía $(5.2 \%)$ y exhibicionismo (4.7\%). Al analizar la distribución de estas prevalencias en ambos grupos, se notaron diferencias estadísticas significativas en la proporción de adolescentes involucrados en las modalidades de exhibicionismo; la cual para la muestra de adolescentes con PAS-VP es de $12.9 \%$, mientras que el grupo con PAS-VM solo alcanza el $4.7 \%$. A su vez, si bien la modalidad más recurrente para ambos grupos es el abuso sexual, se halló un número mayor de adolescentes con PAS-VM en este tipo de PAS (82.9\%) en relación con los adolescentes con PAS-VP, quienes alcanzan el 64.5\% $\left(\mathrm{x}^{2}=22.33, \mathrm{p}<.05\right)$. En las modalidades de violación y exposición a la pornografía, aunque se observaron porcentajes superiores en los adolescentes con PAS-VP, esta diferencia no es significativa; por consiguiente, tienden a presentar proporciones similares en ambas modalidades.

\section{Contexto de PAS}

En gran parte, el contexto donde se producen las PAS es a nivel intrafamiliar con un $79.7 \%$, pues solo el $20.3 \%$ de las PAS sucede en otro tipo de contextos, tales como establecimientos educacionales y espacios públicos (extrafamiliares). Al comparar los dos grupos de adolescentes, se encontraron diferencias significativas $\left(\mathrm{x}^{2}=13.2, \mathrm{p}<.05\right)$ que dan cuenta de que el grupo con PAS-VM tendría una mayor proporción de PAS a nivel 
intrafamiliar, con un $98.8 \%$, mientras que en los adolescentes con PAS-VP tienden a predominar las PAS en otros contextos, con el $72.6 \%$.

\section{Sexo de la víctima}

En la muestra total se apreció un mayor porcentaje de víctimas hombres $(55.2 \%)$ que mujeres (44.8\%). Al comparar ambos grupos, se concluyó que en los adolescentes con PAS-VM existe similar proporción de víctimas mujeres (50.6\%) y hombres (49.4\%); no obstante, se ha evidenciado una diferencia significativa $\left(\mathrm{x}^{2}=10.37, \mathrm{p}<.05\right)$, dado que en el grupo conformado por adolescentes PAS-VP existe una mayor inclinación hacia la elección de víctimas de sexo masculino ( $71 \%)$, en comparación con las de sexo femenino (29\%).

\section{Relación ofensor-víctima}

En la muestra total, la gran mayoría de los adolescentes ofende sexualmente a víctimas conocidas ( $81.5 \%$ ), mientras que solo el $18.5 \%$ lo hace con víctimas desconocidas. Al analizar ambos grupos, si bien los adolescentes con PAS-VM y PAS-VP siguen la tendencia de la muestra general, alcanzan diferencias significativas $\left(X^{2}=19.27, p<.05\right)$, en tanto el grupo con PAS-VP cuenta con una mayor proporción de víctimas desconocidas (37.1\%), si se le compara con el grupo con PAS-VM, en el que estas ascienden solo al $11.8 \%$.

\section{Comisión de otros tipos de delitos}

Se registraron diferencias significativas entre ambos grupos, respecto a los ingresos en programas de responsabilidad penal adolescente, por la imputación o condena de un delito distinto a la PAS $\left(\mathrm{X}^{2}=26.9^{*}, \mathrm{p}<.05\right)$ y se constató que la mayor parte de estos casos se concentra en el grupo con PAS-VP (19.4\%). A modo de resumen, la tabla 5 recoge los resultados de las variables criminológicas descritas en este apartado. 


\section{Tabla 5}

Síntesis de características criminológicas de adolescentes con PAS-VM y PAS-VP

\begin{tabular}{|c|c|c|c|c|c|}
\hline Variable & $\begin{array}{c}\text { Total }(n=232) \\
\bar{X} / \%\end{array}$ & $\begin{array}{c}\text { PAS-VM }(n=170) \\
\bar{X} / \%\end{array}$ & $\begin{array}{c}\text { PAS-VP }(n=62) \\
\bar{X} / \%\end{array}$ & $t \circ X^{2}$ & doV \\
\hline Promedio edad evento abusivo & 13.3 & 13.5 & 13.1 & $t=0.122$ & 0.23 \\
\hline Promedio número de víctimas & 1.3 & 1.1 & 1.5 & $t=1.152$ & 0.21 \\
\hline \multicolumn{6}{|l|}{ Tipo de PAS } \\
\hline Abuso sexual & 78 & 82.9 & 64.5 & \multirow{4}{*}{$X^{2}=22.33^{\star}$} & \\
\hline Violación & 12.1 & 10.6 & 16.1 & & \\
\hline Exposición a pornografía & 5.2 & 4.7 & 6.5 & & 0.43 \\
\hline Exhibicionismo & 4.7 & 1.8 & 12.9 & & \\
\hline \multicolumn{6}{|l|}{ Contexto de PAS } \\
\hline Familiar & 79.7 & 98.8 & 27.4 & \multirow{2}{*}{$X^{2}=38.87^{\star}$} & \multirow[t]{2}{*}{0.53} \\
\hline Extrafamiliar & 20.3 & 1.2 & 72.6 & & \\
\hline \multicolumn{6}{|l|}{ Sexo víctima } \\
\hline Mujer & 44.8 & 50.6 & 29 & \multirow{2}{*}{$X^{2}=10.37^{\star}$} & \multirow[t]{2}{*}{0.41} \\
\hline Hombre & 55.2 & 49.4 & 71 & & \\
\hline \multicolumn{6}{|l|}{ Relación ofensor-víctima } \\
\hline Conocida & 81.5 & 88.2 & 62.9 & \multirow{2}{*}{$X^{2}=19.27^{\star}$} & \multirow[t]{2}{*}{0.55} \\
\hline Desconocida & 18.5 & 11.8 & 37.1 & & \\
\hline \multicolumn{6}{|l|}{ Otros delitos RPA } \\
\hline Sí & 6 & 1.2 & 19.4 & \multirow{2}{*}{$x^{2}=26.9^{*}$} & \multirow{2}{*}{0.35} \\
\hline No & 94 & 98.8 & 80.6 & & \\
\hline
\end{tabular}

Nota: RPA: Ley n. ${ }^{\circ} 20084$ de Responsabilidad Penal Adolescente; * p $<0.05$

\section{Discusión}

Los resultados obtenidos para la muestra estudiada revelaron diferencias significativas entre ambos grupos en 10 de las 27 variables analizadas. Estas indicaron que los adolescentes con PAS-VM presentarían menos dificultades a nivel escolar, menor asociación a otro tipo de comportamientos delictivos, similar proporción de víctimas mujeres y hombres y PAS perpetradas preferentemente contra víctimas conocidas y en un contexto intrafamiliar. Por su parte, los adolescentes con PAS-VP evidencian de manera significativa mayores problemas en su trayectoria educativa, mayor frecuencia de otros tipos de comportamientos delictivos, mayor proporción de víctimas masculinas y predilección 
por víctimas conocidas, en tanto que los hechos de PAS ocurren con mayor recurrencia en contextos extrafamiliares.

En general, estos hallazgos siguen la tendencia de otros estudios publicados a nivel internacional, por ejemplo, en cuanto a la propensión de los adolescentes con PAS-VP a tener más problemas educativos (Leroux et al., 2016; van der Put \& Asscher, 2015) y ser menos proclives a sufrir acoso escolar (Benedicto et al., 2017; Hendriks \& Bijleveld, 2004). Estos datos podrían ser explicados por variables de personalidad de los adolescentes, ámbito en el que algunos estudios han encontrado que los adolescentes con PAS-VM tienen mayor tendencia a internalizar problemas de comportamientos y a mostrar un patrón de funcionamiento inclinado hacia la sumisión y el conformismo; mientras que los adolescentes con PAS-VP revelan mayores problemas de conductas y rasgos antisociales, de modo que es entendible una mayor cantidad de problemas en esta área (Aebi et al., 2012; Glowacz \& Born, 2013; Joyal et al., 2016; Lillard et al., 2019; van der Put \& Asscher, 2015).

En lo concerniente a las experiencias de victimización, se destaca que la totalidad de los participantes del estudio ha sufrido experiencias de victimizaciones a lo largo de su vida. Estas últimas estarían vinculadas preferentemente al contexto familiar, de forma que los espacios familiares podrían ser considerados como estresantes y complejos para los adolescentes. Por otro lado, un hecho llamativo emerge al contrastar los resultados de esta dimensión con los estudios internacionales, debido a que las tasas de victimización encontradas en ambos grupos tienden a ser superiores a las reportadas en investigaciones realizadas en España, Estados Unidos y Canadá (Benedicto et al., 2017; Fanniff \& Kolko, 2012; Leroux et al., 2016). De este modo, se refuerza la evidencia que indica una estrecha relación entre experiencias de esta naturaleza y posibles problemas de comportamiento sexual en edades posteriores (Barra et al., 2017; Boonmann et al., 2016; Dillard \& Beaujolais, 2019; Malvaso et al., 2020; Tsang, 2017; Yoder \& Precht, 2020).

En el caso particular de la victimización sexual previa de los adolescentes con PAS, variable que ha recibido especial atención para explicar los factores etiológicos de las PAS (Johnson \& Knight, 2000; Knight \& Sims-Knight, 2003), los hallazgos del presente estudio mostraron tasas de victimizaciones similares en ambos grupos, las cuales se encumbran sobre el $50 \%$ de los casos. Si bien estas cifras están sobre el $46 \%$ estimado por Seto y Lalumière (2010), las comparaciones deben hacerse con cautela, a causa de las diferencias en los criterios de recolección de información para esta variable, tales como: entrevistas, autoinformes y registros. Conviene aclarar que este antecedente adquiere relevancia a nivel explicativo, no solo en la medida en que respaldaría los modelos basados 
en el aprendizaje, condicionamiento y modelamiento producto de las PAS sufridas (Barbaree \& Marshall, 2006; Burton, 2003), sino debido a que puede ser un foco importante para el tratamiento de estos adolescentes, pues el abuso sexual infantil sería la causa de un desarrollo atípico en la trayectoria de su vida, vinculado con las relaciones con los compañeros y el posterior comportamiento sexual (Seto \& Lalumière, 2010).

En efecto, no son pocas las investigaciones que han descrito que las experiencias de abuso en la infancia (Dillard \& Beaujolais, 2019; Seto \& Lalumière, 2010) y la disfunción familiar (Dillard \& Beaujolais, 2019; Gunby \& Woodhams, 2010; Sitney \& Kaufman, 2019; Yoder et al., 2017), como variables importantes en la etiología de las PAS, requieren de un mayor énfasis en los procesos de prevención y tratamiento. De hecho, en las principales guías de valoración de riesgo de reincidencia para adolescentes con PAS se ha manifestado que la disfunción familiar extrema, los cambios constantes de cuidador y el rechazo parental son indicadores de alto riesgo de reincidencia sexual, por lo que se reafirma la necesidad de establecer objetivos de trabajo en esta área (Miccio-Fonseca, 2006; Rich, 2017; Worling \& Curwen, 2001).

De ahí que este asunto sea clave en los programas de tratamiento ambulatorios para los adolescentes que ejecutan PAS en Chile, en tanto este ratifica la necesidad de consolidar el abordaje de las experiencias de vida adversas en la infancia y la intervención familiar, con el objetivo de favorecer el desistimiento de las PAS y de ampliar la mirada de abordaje más allá del tratamiento de la excitación sexual desviada, el control de impulsos, las habilidades sociales, las distorsiones cognitivas y las conductas agresivas (Prisco, 2015).

Ahora bien, al analizar las características criminológicas, en ambos grupos se destacó una mayor inclinación hacia las víctimas femeninas. Esta diferencia se acentuó en el grupo de adolescentes con PAS-VP, pues alcanzó el 71\%, en tanto que los adolescentes con PAS-VM registraron casi la misma proporción de víctimas hombres y mujeres. En consecuencia, este dato es coherente con la evidencia disponible (Benedicto et al., 2017; Fanniff \& Kolko, 2012; Farrington et al., 2013; Gunby \& Woodhams, 2010; Lillard et al., 2019).

$\mathrm{Si}$ bien estos estudios han argumentado que las diferencias estarían asociadas a factores circunstanciales y de accesibilidad de la víctima, no es menos importante prestar atención a la presencia de intereses y a la orientación sexual hacia hombres o mujeres, dado que la elección indiscriminada de víctimas (hombres y mujeres) y el hecho de haber abusado sexualmente de una víctima masculina, aparecen descritos como factores de riesgo en el registro de las nuevas ofensas sexuales, propuesto en distintas guías de valoración 
de riesgos de reincidencia para adolescentes que presentan PAS (Miccio-Fonseca, 20o6; Prentky \& Righthand, 2003; Rich, 2017; Worling \& Curwen, 2001).

En cuanto al contexto de la PAS y al grado de relación con las víctimas, es posible observar que ambos grupos tienen una mayor habituación a generar PAS en contextos familiares y con víctimas conocidas. Aun cuando no se advierten diferencias estadísticamente significativas, al revisar las frecuencias, dentro del grupo PAS VP se aprecia un mayor número de casos de víctimas desconocidas y de PAS desarrolladas en contextos extrafamiliares; mientras que, en el caso de los adolescentes con PAS VM, se observa mayor cantidad de casos de víctimas conocidas, aspectos que concuerdan con la teoría (Benedicto et al., 2017; Gunby \& Woodhams, 2010; Lillard et al., 2019).

Con relación a las modalidades de PAS, el abuso sexual se presenta en mayor frecuencia en ambos grupos; no obstante, en el grupo PAS-VP se advierte mayor variabilidad en los tipos de PAS, pues se han dado casos de violación y exhibicionismo. Lo anterior sería parcialmente concordante con la literatura que ha estudiado esta variable, pues en algunos estudios no se identificaron diferencias significativas en relación con la modalidad de PAS (Hendriks \& Bijleveld, 2004), mientras que otros sí le atribuyen un mayor índice de severidad en las PAS a aquellos que orientan estas prácticas hacia VP (Lillard et al., 2019).

Por otro lado, el número de adolescentes que presenta ingresos a programas de responsabilidad penal adolescente por otro tipo de delitos (no sexuales) tiende a ser reducido (van Wijk et al., 2007) y se concentra, en mayor medida, en el grupo de adolescentes con PAS-VP. Por ende, este resultado es afín a lo explicado en los estudios internacionales, donde se reportaron mayores problemas de comportamiento para este grupo de adolescentes, una trayectoria de institucionalización más prolongada en el sistema y una mayor vinculación con tribunales de justicia (Aebi et al., 2012; Hunter et al., 2003). En la misma línea, algunos estudios han señalado que los adolescentes que manifiestan otro tipo de comportamiento delictivo, además de la ofensa sexual, tendrían un mayor riesgo de reincidencia y se beneficiarían en menor medida de los tratamientos clínicos, mientras que los adolescentes que solo exhiben ofensas sexuales contarían con más posibilidades de adaptarse a la vida social y tendrían un mayor grado de comportamientos prosociales (Butler \& Seto, 2002).

Los hallazgos de esta investigación refuerzan la hipótesis de la heterogeneidad de los adolescentes con PAS. Este aspecto ha quedado de manifiesto en múltiples estudios, cuyas variables diferenciadoras han sido de diversa índole (Cale et al., 2016; Everhart et al., 2019; Lillard et al., 2019; Ryan \& Otonichar, 2016; Ueda, 2017; Wanklyn et al., 2012; Worling \& 
Langton, 2012) y han revelado la necesidad de aplicar intervenciones diferenciadas para cada subgrupo, según modelos de trabajo que contemplen la evidencia empírica y cuenten con fundamentación teórica y solidez metodológica (Barra et al., 2018; Harris et al., 2016; Prisco, 2015; Yoder \& Ruch, 2015).

Es así como los estudios de caracterización de grupos específicos se asocian a la generación de información actualizada, hasta que logran convertirse en insumos para el diseño de políticas públicas y la elaboración de estrategias de abordaje pertinentes (Lussier et al., 2012; Russell \& Harvey, 2016; Sherman \& Neyroud, 2012). Desde esta perspectiva, existe la posibilidad de que los programas de tratamiento consideren, en la elaboración de planes de intervención, los resultados encontrados en el presente estudio; por ejemplo, en lo que se refiere a los altos niveles de victimizaciones previas sufridas por los adolescentes, la intervención familiar y los elementos criminológicos asociados a la PAS, tales como tipo de ofensa, contexto de ofensa, sexo de víctima y relación ofensor-víctima.

Respecto a las limitaciones de la investigación, es posible señalar que la omisión de variables psicológicas y del nivel de riesgo de reincidencia en el análisis del fenómeno solo permitió describir, en términos generales, a ambos subgrupos. Igualmente, una limitación de la muestra fue la ausencia de adolescentes que se inclinaran hacia víctimas mixtas y el hecho de que los adolescentes partícipes solo obedezcan a sujetos insertos en programas ambulatorios. En concordancia con ello, las futuras investigaciones deberían ampliar el espectro de la muestra a adolescentes con sanciones penales, además de enfocarse en explorar otros elementos relevantes; entre ellos, variables de personalidad, comportamiento social o carrera delictiva, con el objetivo de profundizar en el conocimiento de las características de estos adolescentes.

\section{Referencias}

Aebi, M., Vogt, G., Plattner, B., Steinhausen, H. C., \& Bessler, C. (2012). Offender types and criminality dimensions in male juveniles convicted of sexual offenses. Sexual Abuse: A Journal of Research and Treatment, 24(3), 265-288. https://doi.org/ 10.1177/1079063211420449

Alarcón, P. A., Pérez-Luco Arenas, R. X., Wenger, L. S., Salvo, S. I., \& Chesta, S. A. (2017). Personalidad y gravedad delictiva en adolescentes con conducta antisocial 
persistente.Revista Iberoamericana de Psicología y Salud, 9(1), 58-74. https://doi.org/ 10.23923/j.rips.2018.01.015

Ávila-Navarrete, V. C. (2017). ¿Corresponsabilidad familiar en instituciones de reeducación para adolescentes infractores? Revista Latinoamericana de Ciencias Sociales, Niñez y Juventud, 15(2), 1191-1206.

Barbaree, H., \& Marshall, W. (2006). An introduction to the juvenile sex offenders: Terms, concepts and definitions. En H. Barbaree, \& W. Marshall (Eds.), The juvenile sex offender (pp. 1-18). Guilford Press.

Barra, S., Bessler, C., Landolt, M. A., \& Aebi, M. (2017). Patterns of adverse childhood experiences in juveniles who sexually offended. Sexual Abuse: A Journal of Research and Treatment, 30(7), 803-827. https://doi.org/10.1177/1079063217697135

Barra, S., Bessler, C., Landolt, M. A., \& Aebi, M. (2018). Testing the validity of criminal risk assessment tools in sexually abusive youth. Psychological Assessment, 30(11), 1430-1443. https://doi.org/10.1037/pasoooo59o

Benedicto, C., Roncero, D., \& González, L. (2017). Agresores sexuales juveniles: tipología y perfil psicosocial en función de la edad de sus víctimas. Anuario de Psicología Jurídica, $27(1)$, 33-42. https://doi.org/10.1016/j.apj.2016.05.002

Boonmann, C., Grisso, T., Guy, L. S., Colins, O. F., Mulder, E. A., Vahl, P., Vermeiren, R. R. (2016). Childhood traumatic experiences and mental health problems in sexually offending and non-sexually offending juveniles. Child and Adolescent Psychiatry and Mental Health, 10(1), 45-55. https://doi.org/10.1186/s13034-016-0127-2

Brown, A. (2019). Using latent class analysis to explore subtypes of youth who have committed sexual offenses. Youth Violence and Juvenile Justice, $17(4), 413-430$. https:// doi.org/10.1177/1541204018820578

Burton, D. (2003). Male adolescents: sexual victimization and subsequent sexual abuse. Child and Adolescent Social Work Journal, 2o(4), 277-296. https://doi.org/10.1023/ A:1024556909087

Butler, S., \& Seto, M. C. (2002). Distinguishing two types of adolescent sex offenders. Journal of the American Academy of Child \& Adolescent Psychiatry, 41(1), 83-90. https://doi.org/10.1097/00004583-200201000-00015

Caldwell, M. (2016). Quantifying the decline in juvenile sexual recidivism rates. Psychology, Public Policy, and Law, 22(4), 414-426. https://doi.org/10.1037/lawooooog4

Cale, J., Smallbone, S., Rayment-McHugh, S., \& Dowling, C. (2016). Offense trajectories, the unfolding of sexual and non-sexual criminal activity, and sex offense characteristics 
of adolescent sex offenders. Sexual Abuse: A Journal of Research and Treatment, 28(8), 791-812. https://doi.org/10.1177/1079063215580968

Chaffin, M. (2008). Our minds are made up-don't confuse us with the facts: Commentary on policies concerning children with sexual behavior problems and juvenile sex offenders. Child Maltreatment, 13(2), 110-121. https://doi.org/10.1177/1077559508314510

Dillard, R., \& Beaujolais, B. (2019). Trauma and adolescents who engage in sexually abusive behavior: a review of the literature. Journal of Child Sexual Abuse, 28(6), 629-648. https://doi.org/10.1080/10538712.2019.1598528

Escobar-Pérez, J., \& Cuervo-Martínez, Á. (2008). Validez de contenido y juicio de expertos: una aproximación a su utilización. Avances en Medición, 6(1), 27-36.

Everhart, J. L., Larsen, J. L., Thompson, K., Cyperski, M., \& Burkhart, B. R. (2019). Heterogeneity in male adolescents with illegal sexual behavior: a latent profile approach to classification. Sexual Abuse: A Journal of Research and Treatment, $31(7)$, 789-811. https://doi.org/10.1177/1079063218784554

Fanniff, A. M., \& Kolko, D. J. (2012). Victim age-based subtypes of juveniles adjudicated for sexual offenses: Comparisons across domains in an outpatient sample. Sexual Abuse: A Journal of Research and Treatment, 24(3), 224-264. https://doi.org/ 10.1177/1079063211416516

Farrington, D., Piquero, A., \& Jennings, W. (2013). Offending from childhood to late middle age. Springer. https://doi.org/10.1007/978-1-4614-6105-0

Finkelhor, D., Ormrod, R., \& Chaffin, M. (2009). Juveniles who commit sex offenses against minors. https://bit.ly/37JgiWT

Glowacz, F., \& Born, M. (2013). Do adolescent child abusers, peer abusers, and non-sex offenders have different personality profiles? European Child and Adolescent Psychiatry, $22(2), 117-125$. https://doi.org/10.1007/s00787-012-0333-2

Gunby, C., \& Woodhams, J. (2010). Sexually deviant juveniles: comparisons between the offender and offence characteristics of child abusers and peer abusers. Psychology, Crime \& Law, 16(1-2), 47-64. https://doi.org/10.1080/10683160802621966

Harris, A., Walfield, S., Shields, R., \& Letourneau, E. (2016). Collateral consequences of juvenile sex offender registration and notification: Results from a survey of treatment providers. Sexual Abuse: Journal of Research and Treatment, 28(8), 770-79o. https:// doi.org/10.1177/1079063215574004

Hendriks, J., \& Bijleveld, C. C. (2004). Juvenile sexual delinquents: Contrasting child abusers with peer abusers. Criminal Behaviour and Mental Health, 14(4), 238-250. https://doi.org/10.1002/cbm.591 
Höing, M., Jonker, M., \& van Berlo, W. (2010). Juvenile sex offenders in a Dutch mandatory educational programme: Subtypes and characteristics. Journal of Sexual Aggression, 16(3), 332-346. https://doi.org/10.1080/13552600903350991

Hunter, J. A., Figueredo, A. J., Malamuth, N. M., \& Becker, J. V. (2003). Juvenile sex offenders: toward the development of a typology. Sexual Abuse: A Journal of Research and Treatment, 15(1), 27-48. https://doi.org/10.1177/107906320301500103

Jenkins, C. S., Grimm, J. R., Knight, E., van Dooren, S., Ciesar, E., \& Reid-Quiñones, K. (2020). Preliminary findings of problematic sexual behavior-cognitive-behavioral therapy for adolescents in an outpatient treatment setting. Child Abuse and Neglect, (105), 104428. https://doi.org/10.1016/j.chiabu.2020.104428

Johnson, G., \& Knight, R. (2000). Developmental antecedents of sexual coercion in juvenile sexual offenders. Sexual Abuse: A Journal of Research and Treatment, 12(3), 165-178. https://doi.org/10.1177/107906320001200301

Joyal, C., Carpentier, J., \& Martin, C. (2016). Discriminant factors for adolescent sexual offending: on the usefulness of considering both victim age and sibling incest. Child Abuse and Neglect, (54), 10-22. https://doi.org/10.1016/j.chiabu.2016.01.00

Keelan, C. M., \& Fremouw, W. J. (2013). Child versus peer/adult offenders: a critical review of the juvenile sex offender literature. Aggression and Violent Behavior, 18(6), 732-744. https://doi.org/10.1016/j.avb.2013.07.026

Knight, R. A., \& Sims-Knight, J. E. (2003). The developmental antecedents of sexual coercion against women: testing alternative hypotheses with structural equation modeling. Annals of the New York Academy of Sciences, 989(1), 72-85. https://doi.org/ 10.1111/j.1749-6632.2003.tbo7294.x

Komatsu, A., \& Bazon, M. (2015). Caracterização de adolescentes do sexo masculino em relação a comportamentos antissociais. Revista Latinoamericana de Ciencias Sociales, Niñez y Juventud, 13(2), 725-735.

Krause, C., Roth, A., Landolt, M. A., Bessler, C., \& Aebi, M. (2020). Validity of risk assessment instruments among juveniles who sexually offended: Victim age matters. Sexual Abuse: A Journal of Research and Treatment, 15(1), 1-27. https://doi.org/ 10.1177/1079063220910719

Leroux, E. J., Pullman, L. E., Motayne, G., \& Seto, M. C. (2016). Victim age and the generalist versus specialist distinction in adolescent sexual offending. Sexual Abuse: A Journal of Research and Treatment, 28(2), 79-95. https://doi.org/10.1177/1079063214535814 
Lillard, C. M., Cooper-Lehki, C., Fremouw, W. J., \& DiSciullo, V. A. (2019). Differences in psychosexual development among child, peer, and mixed juvenile sex offenders. Journal of Forensic Sciences, 65(2), 526-534. https://doi.org/10.1111/1556-4029.14194

Lussier, P. (2017). Juvenile sex offending through a developmental life course criminology perspective: An agenda for policy and research. Sexual Abuse: A Journal of Research and Treatment, 29(1), 51-80. https://doi.org/10.1177/1079063215580966

Lussier, P., \& Blokland, A. (2014). The adolescence-adulthood transition and Robins's continuity paradox: Criminal career patterns of juvenile and adult sex offenders in a prospective longitudinal birth cohort study. Journal of Criminal Justice, 42(2), 153-163. https://doi.org/10.1016/j.jcrimjus.2013.07.004

Lussier, P., van den Berg, C., Bijleveld, C., \& Hendriks, J. (2012). A developmental taxonomy of juvenile sex offenders for theory, research, and prevention the adolescentlimited and the high-rate slow desister. Criminal Justice and Behavior, 39(12), 1559-1581. https://doi.org/10.1177/0093854812455739

Malvaso, C. G., Proeve, M., Delfabbro, P., \& Cale, J. (2020). Characteristics of children with problem sexual behaviour and adolescent perpetrators of sexual abuse: $\mathrm{A}$ systematic review. Journal of Sexual Aggression, 26(1), 36-61. https://doi.org/ 10.1080/13552600.2019.1651914

Miccio-Fonseca, L. C. (2006). Multiplex empirically guided inventory of ecological aggregates for assessing sexually abusive children and adolescents (MEGA\&). https://bit.ly/37FtDQc

Ministerio Público. (2019). Boletín estadístico anual. Enero-diciembre 2018. https://bit.ly/ $37 \mathrm{XU}_{2} \mathrm{ZJ}$

Muñiz, J., \& Fonseca-Pedrero, E. (2019). Diez pasos para la construcción de un test. Psicothema, 31(1), 7-16. https://doi.org/10.7334/psicothema2018.291

Oxnam, P., \& Vess, J. (2008). A typology of adolescent sexual offenders: Millon Adolescent Clinical Inventory profiles, developmental factors, and offense characteristics. Journal of Forensic Psychiatry \& Psychology, 19(2), 228-242. https://doi.org/ 10.1080/14789940701694452

Pérez-Luco, R., Alarcón, P., Zambrano, A., Alarcón, M., Chesta, S., \& Wenger, L. (2017). Taxonomía de la delincuencia adolescente con base en evidencia chilena. En C. Bringas, \& M. Novo, Psicología jurídica, conocimiento y práctica (pp. 249-268). Sociedad Española de Psicología Jurídica y Forense.

Prentky, R., \& Righthand, S. (2003). Juvenile Sex Offender Assessment Protocol-II (J-Soap-II) Manual. https://bit.ly/3gkBtT4 
Prisco, R. (2015). Parental involvement in juvenile sex offender treatment: Requiring a role as informed supervisor. Family Court Review, 53(3), 487-503. https://doi.org/ 10.1111/fcre.12169

Rich, P. (2017). Juvenile Risk Assessment Tool Version 4 (J-RAT). https://bit.ly/2Lh287W

Russell, H., \& Harvey, J. (2016). Working with adolescents who display sexually harmful behaviour. Journal of Children's Services, 11(3), 191-203. https://doi.org/10.1108/ JCS-07-2015-0022

Ryan, E. P., \& Otonichar, J. M. (2016). Juvenile sex offenders. Current Psychiatry Reports, 18(67), 1-10. https://doi.org/10.1007/s11920-016-0706-1

Salazar-Muñoz, M., Álvarez-Vivar, L., \& Pérez-Luco Arenas, R. (2016). Instrumentos para la valoración del riesgo de violencia sexual en ofensores sexuales adolescentes: evidencias de validez en países de América Latina. Revista Criminalidad, 58(3), 87-99.

Seto, M. C., \& Lalumière, M. L. (2010). What is so special about male adolescent sexual offending? A review and test of explanations through meta-analysis. Psychological Bulletin, 136(4), 526-575. https://doi.org/10.1037/a0019700

Sherman, L., \& Neyroud, P. (2012). Offender-desistance policing and the sword of Damocles. Civitas.

Short, J. L. (2018). Between-group differences among victim age-based typologies of juvenile sexual offenders [Tesis doctoral no publicada]. Walden University.

Sitney, M., \& Kaufman, K. (2019). The impact of disrupted caregiving for juvenile sexual offenders. Journal of Sexual Aggression, 26(2), 274-287. https://doi.org/ 10.1080/13552600.2019.1618933

Stevens, P., Hutchin, K., French, L., \& Craissati, J. (2013). Developmental and offence-related characteristics of different types of adolescent sex offender: A community sample. Journal of Sexual Aggression, 19(2), 138-157. https://doi.org/10.1080/13552600.2011.645889

ter Beek, E., van der Rijken, R. E., Kuiper, C. H., Hendriks, J., \& Stams, G. J. (2018). The allocation of sexually transgressive juveniles to intensive specialized treatment: An assessment of the application of RNR principles. International Journal of Offender Therapy and Comparative Criminology, 62(5), 1179-1200. https://doi.org/10.1177/0306624X16674684

Tsang, S. (2017). Troubled or traumatized youth? The relations between psychopathy, violence exposure, posttraumatic stress disorder, and antisocial behavior among juvenile offenders. Journal of Aggression, Maltreatment and Trauma, 27(2), 164-178. https://doi.org/10.1080/10926771.2017.1372541 
Ueda, M. (2017). Developmental risk factors of juvenile sex offenders by victim age: An implication for specialized treatment programs. Aggression and Violent Behavior, (37), 122-128. https://doi.org/10.1016/j.avb.2017.09.006

van der Put, C., \& Asscher, J. (2015). Protective factors in male adolescents with a history of sexual and/or violent offending: A comparison between three subgroups. Sexual Abuse: Journal of Research and Treatment, 27(1), 109-126. https://doi.org/ 10.1177/1079063214549259

van Wijk, A., Mali, B., Bullens, R., \& Vermeiren, R. (2007). Criminal profiles of violent juvenile sex and violent juvenile non sex offenders: An explorative longitudinal study. Journal of Interpersonal Violence, 22(10), 1340-1355. https://doi.org/ 10.1177/0886260507304802

Wanklyn, S. G., Ward, A. K., Cormier, N. S., Day, D. M., \& Newman, J. E. (2012). Can we distinguish juvenile violent sex offenders, violent non-sex offenders, and versatile violent sex offenders based on childhood risk factors? Journal of Interpersonal Violence, $27(11), 2128-2143$. https://doi.org/10.1177/0886260511432153

Worling, J., \& Curwen, T. (2001). Estimate of risk of adolescent sexual offense recidivism. En M. Calder, H. Hanks, K. Epps, B. Print, T. Morrison, \& J. Henniker, Juveniles and children who sexually abuse: Frameworks for assessment (pp. 372-397). Russell House.

Worling, J., \& Langton, C. M. (2012). Assessment and treatment of adolescents who sexually offend: Clinical issues and implications for secure settings. Criminal Justice and Behavior, 39(6), 814-841. https://doi.org/10.1177/0093854812439378

Yoder, J., Dillard, R., \& Stehlik, L. (2017). Disparate reports of stress and family relations between youth who commit sexual crimes and their caregivers. Journal of Sexual Aggression, 24(1), 114-124. https://doi.org/10.1080/13552600.2017.1372938

Yoder, J., \& Precht, M. (2020). Victimization experiences and executive dysfunction as discriminating risk indicators for youth offender typologies. International Journal of Offender Therapy and Comparative Criminology, 64(1), 63-82. https://doi.org/ 10.1177/0306624X19865185

Yoder, J., \& Ruch, D. (2015). Youth who have sexually offended: Using strengths and rapport to engage families in treatment. Journal of Child and Family Studies, 24(9), 2521-2531. https://doi.org/10.1007/s10826-014-0054-x 